\title{
CERTAIN INTEGRALS INVOLVING LOGARITHMIC AND EXPONENTIAL FUNCTIONS
}

\author{
M. ASLAM CHAUDHRY and M. AHMAD \\ Department of Mathematical Sciences \\ King Fahd University of Petroleum and Minerals \\ Dhahran, Saudi Arabia \\ (Received September 20, 1991 and in revised form May 5, 1993)
}

ABSTRACT. In this paper we have evaluated the integrals

$$
\int_{0}^{\infty} x^{n-1} \ln x \exp \left(-a x-b x^{-1}\right) d x
$$

and

$$
\int_{0}^{\infty} x^{n-2}\left(a x^{2}-b\right)(\ln x)^{2} \exp \left(-a x-b x^{-1}\right) d x
$$

for all $n=1,2,3, \ldots$. Some applications of the results are discussed and an open problem is posed.

KEY WORDS AND PHRASES. Macdonald functions, logarithmic and exponential function, statistical inference theory.

1991 AMS SUBJECT CLASSIFICATION: 33-A-10.

\section{INTRODUCTION.}

The integrals

$$
\int_{0}^{\infty} x^{n-1} \ln x \exp \left(-a x-b x^{-1}\right) d x, \quad a>0, b>0
$$

and

$$
\begin{array}{r}
\int_{0}^{\infty} x^{n-2}\left(a x^{2}-b\right)(\ln x)^{2} \exp \left(-a x-b x^{-1}\right) d x \\
a>0, b>0, \quad(n=1,2,3, \ldots)
\end{array}
$$

arise in statistical inference theory when Frechet and other distributions are applied to solve important problems related to ocean engineering technology, resource management, and weather phenomena such as estimation and forecasting of wind velocity, flood, rainfall, etc. See $[3]$ and $[6,7,8]$.

The closed form solutions of the integrals (1) and (2) are not known for any $n=1,2,3, \ldots$. Some numerical techniques are used to evaluate these integrals $[2,8]$. We have evaluated these integrals $(1)$ and (2). Some special cases of the results are also discussed. 


\section{LEMMA.}

For $n=0,1,2,3, \ldots$

$$
\begin{aligned}
& \left(\frac{-d}{d x}\right)^{n}\left\{\ln (b / x) K_{0}(2 \sqrt{b x})\right\} \\
& =\frac{1}{x^{n}}\left[(b x)^{n / 2} \ln (b / x) K_{n}(2 \sqrt{b x})+(n !) \sum_{\jmath=1}^{n} \frac{(b x)^{(n-\jmath) / 2} K_{n-\jmath}(2 \sqrt{b x})}{\jmath(n-\jmath) !}\right] \\
& \quad(a>0, \quad x>0) .
\end{aligned}
$$

PROOF. This follows from the Leibnitz rule of differentiation and from the recursion formulas $[5$, p 970$]$.

3. THEOREM.

$$
\begin{aligned}
& \int_{0}^{\infty} x^{n-1} \ln x \exp \left(-a x-b x^{-1}\right) d x \\
&=\frac{1}{a^{n}}\left[(a b)^{n / 2} \ln (b / a) K_{n}(2 \sqrt{a b})+(n !) \sum_{j=1}^{n} \frac{(a b)^{(n-\jmath) / 2} K_{n-\jmath}(2 \sqrt{a b})}{j(n-\jmath) !}\right] \\
&(a>0, b>0, n=0,1,2,3, \ldots) .
\end{aligned}
$$

PROOF. It is known that $[5, \mathrm{p} 313]$

$$
\begin{aligned}
\int_{0}^{\infty} x^{\alpha-1} \exp \left(-a x-b x^{-1}\right) d x=2(b / a)^{\alpha / 2} K_{\alpha}(2 \sqrt{a b}) & \\
a>0, b & >0 .
\end{aligned}
$$

Performing formal differentiation with respect to the parameter $\alpha$ we obtain the equality

$$
\begin{array}{r}
\int_{0}^{\infty} x^{\alpha-1} \ln x \exp \left(-a x-b x^{-1}\right) d x=2(b / a)^{\alpha / 2} \times \\
\times\left[\frac{1}{2} \ln (b / a) K_{\alpha}(2 \sqrt{a b})+\frac{\partial}{\partial \alpha} K_{\alpha}(2 \sqrt{a b})\right] .
\end{array}
$$

The process of the formal differentiation is justified [4, pp. 427-448].

Using the integral representation [5, p. 358] for the Macdonald function $K_{\alpha}$ we get

$$
\frac{\partial}{\partial \alpha}\left[K_{\alpha}(z)\right]=\int_{0}^{\infty} t e^{-z \cosh (t)} \sinh (\alpha t) d t
$$

which implies

$$
\frac{\partial}{\partial \alpha}\left[K_{\alpha}(z)\right]_{\alpha=0}=0
$$

Letting $\alpha \rightarrow 0$ in (6) and using (8) we get

$$
\begin{array}{r}
\int_{0}^{\infty} \frac{1}{x} \ln x \exp \left(-a x-b x^{-1}\right) d x=\ln (b / a) K_{0}(2 \sqrt{a b}), \\
(a>0, b>0) .
\end{array}
$$

We can rewrite (9) in the operational form as follows:

$$
L\left\{\frac{\ln x}{x} \exp \left(-b x^{-1}\right) ; a\right\}=\ln (b / a) K_{0}^{\prime}(2 \sqrt{a b}),
$$

where $L$ is the Laplace transform operator [1]. 
By using the lemma and property [1]

$$
L\left\{x^{n} f(x) ; a\right\}=\left(-\frac{d}{d a}\right)^{n} F(a) \quad(n=1,2,3, \ldots)
$$

of the Laplace transformation we get the result from (9) and (11).

COROLLARY 1. See [5, p. 577]

$$
\begin{aligned}
& \int_{0}^{\infty} \exp (-\mu(x / c+c / x)) \frac{\ln x}{x} d x=2(\ln c) K_{0}(2 \mu) \\
&(c>0, \mu>0) .
\end{aligned}
$$

PROOF. This follows from (9) when we take $a=\mu / c$ and $b=\mu c$.

COROLLARY 2.

$$
\int_{-\infty}^{\infty} t \exp (\beta t-2 a \cosh \beta t) d t=1 / a \beta^{2} K_{0}(2 a)
$$

$(a>0, \beta>0)$.

PROOF. This follows from (4) when we take $n=1, b=a$ and use the transformation $x=$ $e^{\beta t}, \beta>0$.

COROLLARY 3. For all $n=0,1,2,3, \ldots$ and $a>0, b>0$,

$$
\begin{aligned}
\int_{0}^{\infty} x^{n-2}\left(a x^{2}-b\right)(\ln x)^{2} \exp \left(-a x-b x^{-1}\right) d x \\
\quad=\frac{2}{a^{n}}\left[(b a)^{n / 2} \ln (b / a) K_{n}(2 \sqrt{a b})+n ! \sum_{\jmath=1}^{n}(a b)^{n-\jmath) / 2} \frac{K_{n-\jmath}(2 \sqrt{a b})}{j(n-j) !}\right]
\end{aligned}
$$

PROOF. This follows by applying integration by parts to the integral in (4).

In particular for $n=0, a=\mu / c, b=\mu c, \mu>0, c>0$ in (12), we get

$$
\begin{gathered}
\int_{0}^{\infty} \frac{(\ln x)^{2}}{x^{2}}\left(x^{2}-1\right) \exp (-\mu(x / c+c / x)) d x=4 \ln c K_{0}(2 \mu) \\
(\mu>0, c>0) .
\end{gathered}
$$

We state here an open problem. The solution to the problem will have far-reaching consequences in statistical inference theory. It should be noted that the solution to the problem is not known even for $n=2$ and for any value of $\alpha$.

\section{STATEMENT OF THE OPEN PROBLEM.}

$$
\text { Evaluate } \int_{0}^{\infty}(\ln x)^{n} x^{\alpha-1} \exp \left(-a x-b x^{-1}\right) d x \quad(a>0, b>0, n \geq 2) .
$$

ACKNOWLEDGEMENTS. The authors are indebted to the KFUPM for the excellent research facilities. The helpful comments made by the referee contributed to the improvement of the paper and are appreciated. 


\section{REFERENCES}

1. ERDELYI. A., et al., Tables of Integral Transforms, vol. I, (McGraw-Hill, 1954).

2. CHAUDHRY, M.A. and AHMAD, M. On a probability function useful in size modeling. Accepted for publication in Canadian Journal of Forest Research (1992).

3. FISHER, R.A. and TIPPETT, L.H. Limiting forms of the frequency distributions of the largest or smallest member of a sample, Proceedings of the Cambridge Philosophical Society, $24(1928), 180-190$.

4. BUDAK, B.M. and FOMIN, S.V. Multiple Integrals, Field Theory and Series, (Mir Publishers, 1978).

5. GRADSHTEYN, I.S. and RYZHIK, I.M. Tables of Integrals, Series, and Products (Academic Press, 1980).

6. GUMBEL, E.J. A quick estimation of the parameters of Frechet distribution, Review of the International Statistical Institute, 33(3), (1965), 349-363.

7. SINGH, N.P. and SINGH, K.P. Estimation of Frechet distribution parameters by joint distribution

of ' $m$ ' extremes, Statistica, Anno, L., 1(1990), 59-68.

8. SINGH, N.P. Maximum likelihood estimation of Frechet distribution parameters, Journal of Statistical Studies, 7(1987), 11-22. 


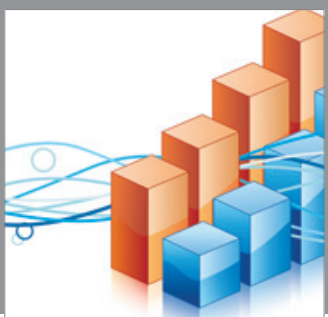

Advances in

Operations Research

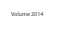

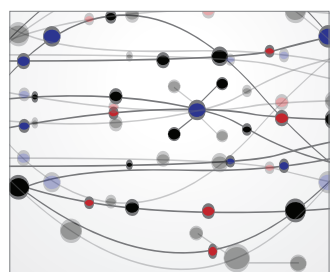

\section{The Scientific} World Journal
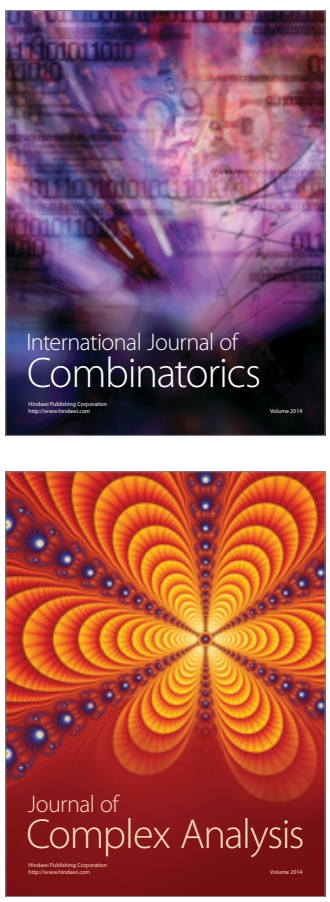

International Journal of

Mathematics and

Mathematical

Sciences
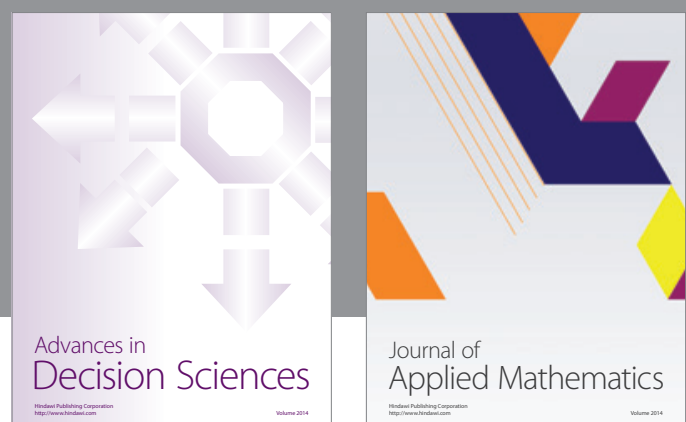

Journal of

Applied Mathematics
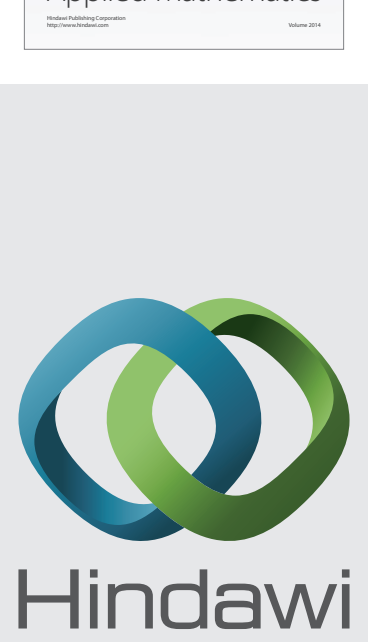

Submit your manuscripts at http://www.hindawi.com
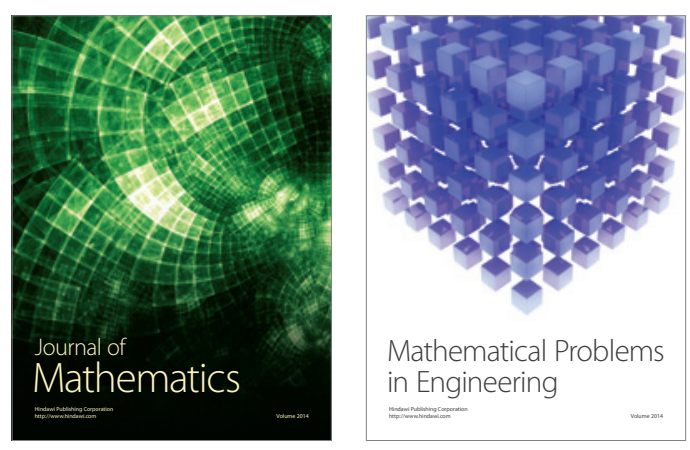

Mathematical Problems in Engineering
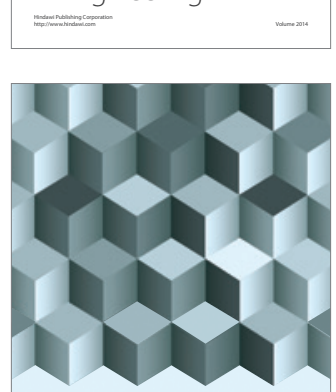

Journal of

Function Spaces
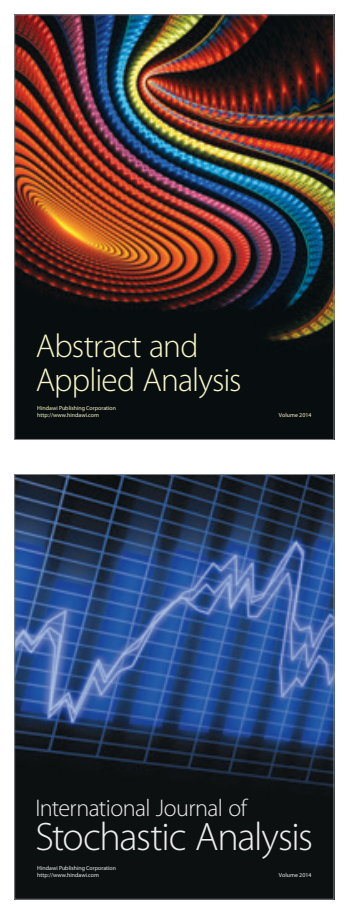

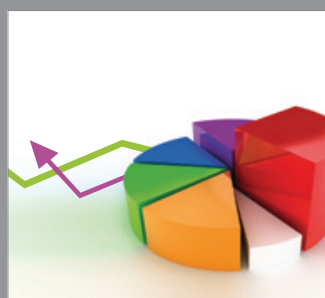

ournal of

Probability and Statistics

Promensencen
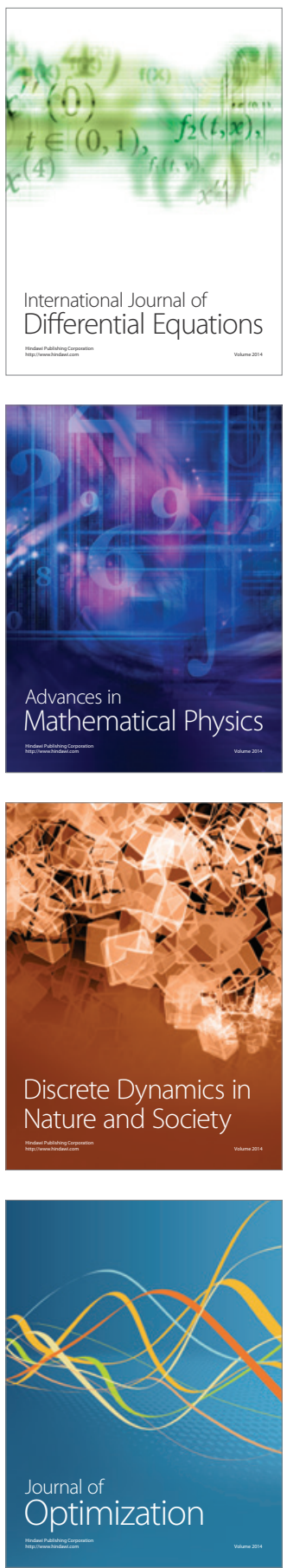